\title{
THE BEHAVIOR OF A BOUNDARY VALUE PROBLEM AS THE INTERVAL BECOMES INFINITE*
}

BY

W. E. MULNE

The boundary value problems for linear self-adjoint differential equations of the second order with homogeneous linear boundary conditions at the ends of a finite interval have been extensively studied and the principal facts are well known. In this connection a problem of some interest arises if the ends of the interval at which the boundary conditions apply are allowed to recede to $-\infty$ and $+\infty$. The aim of this paper is to investigate the behavior of characteristic numbers, characteristic solutions, and oscillation properties, as the interval becomes infinite. A closely related problem for the differential equation

$$
(d / d x)(p(x) d u / d x)+(\lambda-q(x)) u=0
$$

has been solved by Weyl $\dagger$ and further studied by Hilb $\ddagger$ and Gray. $\S$

In this paper some interesting results are obtained for the equation

$$
d^{2} u / d x^{2}+G(x, \lambda) u=0
$$

and are set forth in Theorems I and II.

It is planned to treat the degree of convergence of certain associated expansions for the infinite interval in a subsequent paper.

1. The differential equation under investigation is

$$
d^{2} u / d x^{2}+G(x, \lambda)=0 .
$$

The function $G(x, \lambda)$ is assumed to be real and continuous and to possess a positive partial derivative with respect to $\lambda$ for all real values of $x$ and $\lambda$. It is further assumed that

$$
\lim _{\lambda=-\infty} G(x, \lambda)=-\infty, \quad \lim _{\lambda=+\infty} G(x, \lambda)=+\infty,
$$

and that

$$
\lim _{x= \pm \infty} G(x, \lambda)=-\infty .
$$

\footnotetext{
* Presented to the Society, San Francisco Section, June 2, 1928; received by the editors in December, 1927, and May, 1928.

† Mathematische Annalen, vol. 68 (1910), p. 220, and Göttinger Nachrichten, 1910, p. 442.

$\ddagger$ Mathematische Annalen, vol. 76 (1915), p. 333.

$\$$ American Journal of Mathematics, vol. 50 (1928), p. 431.
} 
These conditions are all satisfied in the important special case

$$
G(x, \lambda)=\lambda-q(x)
$$

provided that $q(x)$ is real and continuous and $\lim q(x)=+\infty$.

Associated with (1) we consider boundary conditions at $x=a$ and $x=b$,

$$
\begin{aligned}
& \alpha_{1} u(a)+\alpha_{2} u^{\prime}(a)+\alpha_{3} u(b)+\alpha_{4} u^{\prime}(b)=0, \\
& \beta_{1} u(a)+\beta_{2} u^{\prime}(a)+\beta_{3} u(b)+\beta_{4} u^{\prime}(b)=0 .
\end{aligned}
$$

It is assumed of course that these conditions are linearly independent. Let the determinant $\alpha_{i} \beta_{j}-\alpha_{j} \beta_{i}$ be denoted by $A_{i j}$, and let $A_{12}=A_{34}$, so that the conditions are self-adjoint. Then there exists* an infinite sequence of values of $\lambda, l_{0}, l_{1}, l_{2}, l_{3}, \cdots$, with limit point at $+\infty$ only, and furnishing solutions of (1) and (4). If we take these values in increasing order of magnitude; counting each double value twice, the solution $u_{n}(x)$ corresponding to $\lambda=l_{n}$ vanishes at least $n$ times and not more than $n+2$ times in the interval $a<x \leqq b$.

Now as $a$ and $b$ recede to $-\infty$ and $+\infty$ respectively, what becomes of the $l_{n}$, the $u_{n}(x)$, and the roots of $u_{n}(x)$ ?

2. In order to answer the foregoing question we must recall some facts concerning the solutions of equation (1), their roots and their behavior at infinity. Multiply (1) by $u(x)$ and integrate from $x_{1}$ to $x_{2}$, integrating the first term by parts. After transposition we have

$$
u\left(x_{2}\right) u^{\prime}\left(x_{2}\right)-u\left(x_{1}\right) u^{\prime}\left(x_{1}\right)=\int_{x_{1}}^{x_{2}} u^{\prime 2}(x) d x-\int_{x_{1}}^{x_{2}} G(x, \lambda) u(x) d x .
$$

From the hypothesis (3) it follows that there exist two numbers $\alpha$ and $\beta$ such that $G(x, \lambda)$ is negative when $x>\beta$ and when $x<\alpha$. Then if $x_{2}>x_{1}>\beta$ the right hand side of (5) is positive, which shows that the product $u(x) u^{\prime}(x)$ does not vanish at both $x_{1}$ and $x_{i}$. Stated Qtherwise, $u(x) u^{\prime}(x)$ does not have more than one root greater than $\beta$ and does not have more than one root less than $\alpha$. The total number of roots of $u(x)$ is therefore finite since the interval between any two consecutive roots is not less than $\pi / M$, where $M^{2}$ is a constant such that $G(x, \lambda) \leqq M^{2}$.

Now it is quite important to show that as $x$ becomes infinite $\dagger$

$$
\lim u^{\prime}(x) / u(x)=\infty
$$

* See for example Birkhoff, these Transactions, vol. 10 (1909), p. 264.

$\dagger$ For the behavior of solutions at infinity see Wiman, Arkiv för Matematik, Astronomi och Fysik, vol. 12, No. 14. 
To see this we note first of all that when $x$ is greater than the greatest root of $u(x)$ the function $R=u^{\prime}(x) / u(x)$ is continuous. Let $N$ be any positive number, as large as we please, and let $x$ be chosen so large that $-G(x, \lambda)$ remains greater than $2 N^{2}$. By differentiation and substitution from (1) we get

$$
d R / d x=-G(x, \lambda)-R^{2},
$$

so that if $R$ ever comes within the interval $-N<R<N$, the derivative $d R / d x$ will be greater than $N^{2}$. Consequently $R$ will increase beyond $N$, and will not return, since $R$ is continuous and $d R / d x$ is positive for $R=N$. Thus (6) is established.

Let the principal solutions of equation (1) at the origin be denoted by $u_{1}(x)$ and $u_{2}(x)$ so that

$$
u_{1}(0)=u_{2}^{\prime}(0)=1, \quad u_{2}(0)=u_{1}^{\prime}(0)=0 .
$$

These solutions satisfy the well known identity

$$
u_{1}(x) u_{2}^{\prime}(x)-u_{2}(x) u_{1}^{\prime}(x)=1 .
$$

Then the general solution of equation (1) can be written

$$
u(x)=C\left(u_{1}^{2}+u_{2}^{2}\right)^{1 / 2} \sin [\phi(x)-\theta],
$$

in which $C$ and $\theta$ are arbitrary constants and $\phi(x)$ is defined by the equation

$$
\phi(x)=\tan ^{-1} u_{2}(x) / u_{1}(x) .
$$

By differentiating (10) and using (8) we get

$$
d \phi / d x=\left[u_{1}^{2}+u_{2}^{2}\right]^{-1},
$$

so that $d \phi / d x$ is positive and $\phi$ is an increasing function of $x$. In view of the fact that $u(x)$ has a finite number of roots, $\phi(x)$ cannot increase indefinitely, and therefore must approach a limit. The same conclusion applies as $x$ becomes negatively infinite. We therefore may define $\phi_{1}$ and $\phi_{2}$ as follows:

$$
\phi_{1}=\lim _{x=-\infty} \phi(x), \quad \phi_{2}=\lim _{x=\infty} \phi(x) .
$$

It is now convenient to define two pairs of independent particular solutions of equation (1) as follows:

$$
\begin{aligned}
V_{1}(x) & =\left(u_{1}^{2}+u_{2}^{2}\right)^{1 / 2} \sin \left[\phi(x)-\phi_{1}\right], \\
W_{1}(x) & =\left(u_{1}^{2}+u_{2}^{2}\right)^{1 / 2} \cos \left[\phi(x)-\phi_{1}\right],
\end{aligned}
$$

and another pair $V_{2}$ and $W_{2}$ similarly defined with $\phi_{2}$ in place of $\phi_{1}$. As $x$ approaches $-\infty$ it can be shown that 


$$
\lim V_{1}(x)=\lim V_{1}^{\prime}(x)=0, \quad \lim W_{1}(x)=\lim W_{1}^{\prime}(x)=\infty,
$$

and as $x$ approaches $+\infty$

$$
\lim V_{2}(x)=\lim V_{2}^{\prime}(x)=0, \quad \lim W_{2}(x)=\lim W_{2}^{\prime}(x)=\infty .
$$

3. It is now necessary to consider how $\phi_{1}$ and $\phi_{2}$ vary with $\lambda$. If $u(x)$ is a solution expressed in the form (9) in which $C$ and $\theta$ are independent of $\lambda$ we may derive in the usual manner* the equation

$$
u^{\prime} \partial u / \partial \lambda-u \partial u^{\prime} / \partial \lambda=\int_{0}^{x}(\partial G / \partial \lambda) u^{2} d x
$$

since at the origin $\partial u / \partial \lambda=\partial u^{\prime} / \partial \lambda=0$ in view of (7). Let $r$ be a root of $u(x)$, so that $-u_{1}(r) \sin \theta+u_{2}(r) \cos \theta=0$. By differentiating this equation with respect to $\lambda$ and making some simplifications by means of (8) and (14) we obtain

$$
\partial r / \partial \lambda=-\left[u_{1}^{2}(r)+u_{2}^{2}(r)\right] \int_{0}^{x}(\partial G / \partial \lambda) u^{2} d x
$$

This shows that as $\lambda$ increases all roots of $u(x)$ move toward the origin. In the same manner if $\lambda$ is constant and $\theta$ varies

$$
\partial r / \partial \theta=u_{1}^{2}(r)+u_{2}^{2}(r),
$$

from which we see that as $\theta$ increases all roots of $u(x)$ move to the right. Finally if $\lambda$ and $\theta$ vary in such a manner as to keep the root $r$ fixed, we get from (15) and (16)

$$
\partial \theta / \partial \lambda=\int_{0}^{r}(\partial G / \partial \lambda) u^{2} d x
$$

We conclude that as $\lambda$ increases $\theta$ also increases when $r$ is positive, but decreases when $r$ is negative. Now at a root of $u(x)$ we have $\theta=\phi(x)+k \pi$ $(k=0, \pm 1, \pm 2, \cdots)$ so that

$$
\partial \phi / \partial \lambda=\int_{0}^{r}(\partial G / \partial \lambda) u^{2} d x .
$$

From this we see that $\phi_{2}$ increases as $\lambda$ increases while $\phi_{1}$ decreases as $\lambda$ increases.

4. Now let $\lambda$ increase from $-\infty$ to $+\infty$ and note the change in the quantity $\phi_{2}-\phi_{1}$. Since there cannot be two roots of $u(x) u^{\prime}(x)$ when $\lambda$ is

* See Sturm, Journal de Mathématiques Pures et Appliquées, vol. 1 (1836), pp. 106-186, especially p. 113. 
large and negative because $G(x, \lambda)$ is negative, it follows that, for such values of $\lambda, \phi_{2}-\phi_{1}<\pi$. But as $\lambda$ increases there will be an infinite sequence of values of $\lambda$ for which the increasing quantity $\phi_{2}-\phi_{1}=\pi, 2 \pi, 3 \pi, \cdots$, since $\phi_{2}-\phi_{1}$ must increase without limit. We may denote these values of $\lambda$ in order of increasing magnitude by $\lambda_{0}, \lambda_{1}, \lambda_{2}, \cdots$. For $\lambda=\lambda_{n}$, it is at once apparent that the solutions $V_{1}(x)$ and $V_{2}(x)$ are identical, except perhaps for sign, and the same is true of $W_{1}(x)$ and $W_{2}(x)$. We may therefore define a solution $U_{n}(x)$ corresponding to $\lambda_{n}$ as follows:

$$
U_{n}(x)=V_{1}(x)= \pm V_{2}(x) \text {, when } \lambda=\lambda_{n} .
$$

We have therefore

$$
\lim _{x= \pm \infty} U_{n}(x)=\lim _{x= \pm \infty} U_{n}^{\prime}(x)=0 .
$$

If we form equation (5) for the solution $U_{n}(x)$ and let $x_{2}$ approach $+\infty$ and $x_{1}$ approach $-\infty$, we see at once from (20) that the integrals

$$
\int_{-\infty}^{+\infty} U_{n}^{\prime 2}(x) d x \text { and } \int_{-\infty}^{+\infty} G(x, \lambda) U_{n}^{2}(x) d x
$$

both converge. From the second of these we may conclude that

also converges.

$$
\int_{-\infty}^{+\infty} U_{n}^{2}(x) d x
$$

Since the number of roots of $U_{n}(x)$ is equal to $k-1$ when $\phi_{2}-\phi_{1}=k \pi$, we see that $U_{n}(x)$ has exactly $n$ roots.

The for egoing conclusions may be summarized as follows:

THEOREM I. There exists an infinite set of critical values of $\lambda, \lambda_{0}, \lambda_{1}$, $\lambda_{2}, \cdots$, with limit point at $+\infty$ only, corresponding to which equation (1) has solutions (unique except for a constant factor) satisfying the conditions

$$
\lim _{x= \pm \infty} U_{n}(x)=\lim _{x= \pm \infty} U_{n}^{\prime}(x)=0 .
$$

The solution $U_{n}(x)$ vanishes $n$ times in the interval $-\infty<x<\infty$, and the integrals

all exist.

$$
\int_{-\infty}^{\infty} U_{n}^{2}(x) d x, \quad \int_{-\infty}^{\infty} U_{n}^{\prime 2}{ }^{2}(x) d x, \text { and } \int_{-\infty}^{\infty} G(x, \lambda) U_{n}^{2}(x) d x
$$

5. We return to the consideration of the conditions (4). The general solution of (1) may be written

$$
u(x)=V_{1}(x) h+W_{1}(x) k,
$$


so that the conditions (4) are equivalent to

$$
\begin{aligned}
{\left[\alpha_{1} V_{1}(a)\right.} & \left.+\alpha_{2} V_{1}^{\prime}(a)+\alpha_{3} V_{1}(b)+\alpha_{4} V_{1}^{\prime}(b)\right] h \\
& +\left[\alpha_{1} W_{1}(a)+\alpha_{2} W_{1}^{\prime}(a)+\alpha_{3} W_{1}(b)+\alpha_{4} W_{1}^{\prime}(b)\right] k=0, \\
{\left[\beta_{1} V_{1}(a)\right.} & \left.+\beta_{2} V_{1}^{\prime}(a)+\beta_{3} V_{1}(b)+\beta_{4} V_{1}^{\prime}(b)\right] h \\
& +\left[\beta_{1} W_{1}(a)+\beta_{2} W_{1}^{\prime}(a)+\beta_{3} W_{1}(b)+\beta_{4} W_{1}^{\prime}(b)\right] k=0 .
\end{aligned}
$$

The determinant of these equations is

$$
\Delta=\left|\begin{array}{c}
{\left[\alpha_{1} V_{1}(a)+\alpha_{2} V_{1}^{\prime}(a)+\alpha_{3} V_{1}(b)+\alpha_{4} V_{1}^{\prime}(b)\right]} \\
{\left[\alpha_{1} W_{1}(a)+\alpha_{2} W_{1}^{\prime}(a)+\alpha_{3} W_{1}(b)+\alpha_{4} W_{1}^{\prime}(b)\right]} \\
{\left[\beta_{1} V_{1}(a)+\beta_{2} V_{1}^{\prime}(a)+\beta_{3} V_{1}(b)+\beta_{4} V_{1}^{\prime}(b)\right]} \\
{\left[\beta_{1} W_{1}(a)+\beta_{2} W_{1}^{\prime}(a)+\beta_{3} W_{1}(b)+\beta_{4} W_{1}^{\prime}(b)\right]}
\end{array}\right|
$$

We shall treat only the case for which $A_{42}$ is not zero, as the modifications to be made when $A_{42}=0$ are sufficiently obvious. Let $\epsilon$ be a positive constant, as small as we please. We may choose $b$ so large that $\left|V_{1}(b) / V_{1}^{\prime}(b)\right|<\epsilon$, and then choose $a$ so large (and negative) that $\left|V_{1}(a)\right|<\epsilon,\left|V_{1}^{\prime}(a)\right|<\epsilon$, $W_{1}(a) / W_{1}^{\prime}(a)<\epsilon,\left|W_{1}(b) / W_{1}^{\prime}(a)\right|<\epsilon,\left|W_{1}^{\prime}(b) / W_{1}^{\prime}(a)\right|<\epsilon$, uniformly with respect to $\lambda$ in an interval $\lambda_{n-1}+\epsilon \leqq \lambda \leqq \lambda_{n}-\epsilon$. Then the determinant may be written

$$
\Delta=V_{1}^{\prime}(b) W_{1}^{\prime}(a)\left[A_{42}+\epsilon E\right],
$$

in which $E$ denotes a function that is bounded for $\lambda$ in the given interval. Since $W_{1}^{\prime}(a)$ does not vanish when $a$ is large and negative and $V_{1}^{\prime}(b)$ does not vanish for $\lambda$ in the given interval when $b$ is large, we see at once that $\Delta$ does not vanish in this interval. But in the next interval $\lambda_{n}+\epsilon \leqq \lambda \leqq \lambda_{n+1}-\epsilon$ the sign is changed and therefore $\Delta$ vanishes between $\lambda_{n}-\epsilon$ and $\lambda_{n}+\epsilon$. Therefore the roots of $\Delta$ approach $\lambda_{n}(n=0,1,2, \cdots)$. Moreover we can easily show that $\Delta$ does not vanish more than once in the interval $\lambda_{n}-\epsilon<\lambda<\lambda_{n}+\epsilon$, so that the characteristic numbers $l_{n}$ of $\$ 1$ are ultimately all simple and $\lim l_{n}=\lambda_{n}$.

From (12), (13), (19), and (20) it will be seen that (21) will be satisfied by taking $h \neq 0, k=0$ when $a$ and $b$ are infinite. Therefore the characteristic functions $u_{n}(x)$ of $\S 1$ approach the functions $U_{n}(x)$. We therefore have

TheOREM II. As the ends of the interval recede to $-\infty$ and $+\infty$ the characteristic numbers all become simple and

$$
\lim l_{n}=\lambda_{n} \quad \lim u_{n}(x)=U_{n}(x) \quad(n=0,1,2, \cdots),
$$

the limits being entirely independent of the boundary conditions (4).

UNIVERSITY OF OREgON, EUGENE. ORE. 